\title{
Identification of chromatin modifying mechanisms in inflammatory macrophages in rheumatoid arthritis
}

Kelly Rooke ${ }^{1}$, Pal Mander ${ }^{2}$, Laurens Kruidenier ${ }^{2}$, Fernando Martinez ${ }^{1}$, Hussein Al-Mossawi ${ }^{1}$, Paul Bowness $^{1}$, Catherine Swales ${ }^{1}$, Raj Prinjha ${ }^{2}$, Udo Oppermann ${ }^{1}$

${ }^{1}$ Botnar Research Centre, NDORMS, Oxford, UK. ${ }^{2}$ EpiNova, GlaxoSmithKline, Stevenage, UK

\begin{abstract}
Background Alterations in epigenetic mechanisms have been implicated in the regulation of proinflammatory cytokine production, and bromodomain-containing proteins (the BET family) may have a specific role in the transcriptional regulation of inflammatory genes. Our objective was to examine specific epigenetic variation between the responses of macrophages derived from RA patients and healthy volunteers, and those derived from inflammatory synovial fluid (SF). Delineation of any epigenetic differences between different macrophage populations may highlight new therapeutic targets in inflammatory disease.
\end{abstract}

\section{Materials and Methods}

A library of small molecule inhibitors, including BET protein inhibitors, was used to identify key epigenetic regulators of pro-inflammatory cytokine production from macrophages. Macrophages were differentiated from the blood monocytes of normal volunteers and RA patients. Synovial fluid derived macrophages were isolated from RA patients undergoing therapeutic arthrocentesis. Cell culture media was supplemented with a small molecule inhibitor, and lipopolysaccharide subsequently used to stimulate inflammatory cytokine production. Cell viability was confirmed using WST-1 assay, cytokine production was measured using an MSD platform and qPCR to assess gene expression. Each experimental condition was performed in triplicate, alongside positive and negative controls. The study was ethically approved and all samples were obtained with informed consent.

\section{Results}

Inhibitors of BET proteins (JQ1, I-BET151 and PFI-1) were the only class of inhibitor to show consistent down-regulation of pro-inflammatory cytokines in both healthy and RA-patient derived macrophages. BET protein inhibitors significantly suppressed the production of TNF $\alpha$ and IL- 6 production from RA blood derived macrophages and IL-6 from corresponding SF-derived macrophages. However, only JQ1 achieved significant inhibition of TNF $\alpha$ production from SF-derived macrophages; it also abrogated LPS-induced cytokine and chemokine mRNA expression in the macrophages of patients with RA.

\section{Conclusions}

This data suggests that the BET proteins are essential regulators of pro-inflammatory cytokine and chemokine production from the macrophages of patients with RA. The differential response to BET inhibition by macrophages derived from blood and synovial fluid of RA patients could indicate alteration in BET protein distribution at pro-inflammatory cytokine promoters, influenced by the chronic inflammatory environment. This study highlights the therapeutic potential of BET inhibitors (particularly JQ1) in RA. 